\title{
A case of an isolated cleft in the posterior mitral leaflet
}

\author{
Hulbert Do · Hiroki Ito · William Jacobs
}

Received: 27 January 2009/Revised: 19 July 2009/Accepted: 22 July 2009/Published online: 18 September 2009

(C) Japanese Society of Echocardiography 2009

A 42-year-old woman complained of palpitation and chest discomfort. A mid-systolic click and faint systolic murmur were auscultated best at the apex. The transthoracic echocardiogram showed the presence of a cleft in the posterior mitral valve leaflet, along with posterior leaflet prolapse and mild mitral regurgitation (MR). The origin and direction of the MR jet suggest that the cleft is the mechanism of the regurgitation. No defect was noted in the anterior leaflet. An isolated cleft in the posterior mitral valve leaflet is an extremely rare congenital malformation, with only five cases being reported in the medical literature [1] (see Figs. 1, 2).

\section{Reference}

1. Amin A, Davis M, Auseon A. Isolated cleft posterior mitral valve leaflet: an uncommon cause of mitral regurgitation. Eur J Echocardiogr. 2008 (Epub ahead of print).

Electronic supplementary material The online version of this article (doi:10.1007/s12574-009-0019-z) contains supplementary material, which is available to authorized users.

\section{H. Do}

Department of Medicine, Loyola University Medical Center, Maywood, IL, USA

\section{H. Ito $(\bowtie) \cdot W$. Jacobs}

Division of Cardiology, Loyola University Medical Center,

EMS Building 6th Floor, 2160 S. First Avenue,

Maywood, IL 60153, USA

e-mail: itohiroki@gmail.com

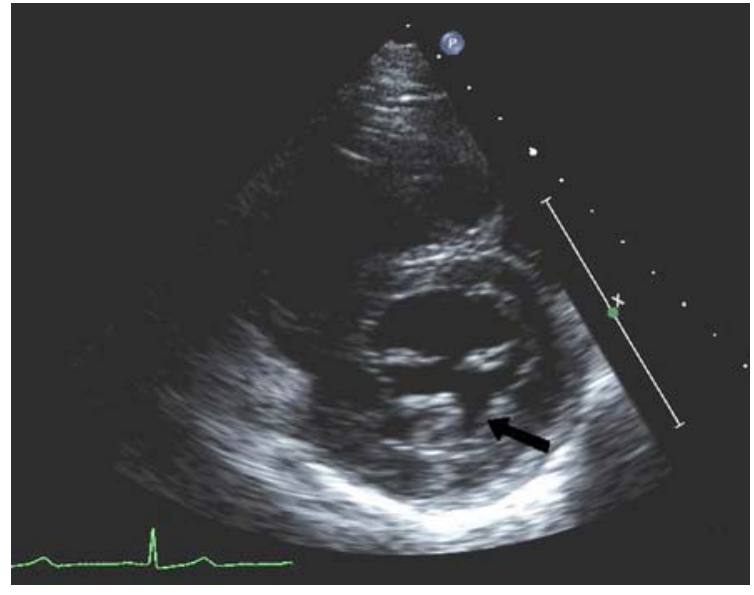

Fig. 1 Parasternal short-axis view showing the presence of a cleft in the posterior mitral valve (indicated by arrow). Please see moving images online for better understanding



Fig. 2 Parasternal long-axis view showing mild mitral regurgitation, which is often seen with posterior mitral valve cleft 